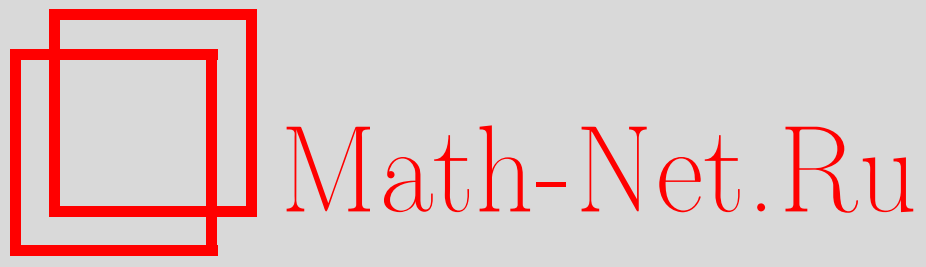

И. Г. Щербак, Некристаллографические группы Кокстера и краевые особенности, Функи. анализ и его прил., 2001, том 35, выпуск 2, 92-94

DOI: https://doi.org/10.4213/faa253

Использование Общероссийского математического портала MathNet.Ru подразумевает, что вы прочитали и согласны с пользовательским соглашением

http://www . mathnet.ru/rus/agreement

Параметры загрузки:

IP : 52.23 .180 .231

26 апреля 2023 г., 18:01:14

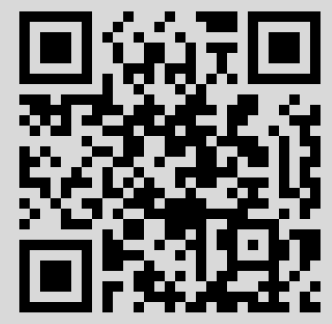


собственных значений оператора $L(\kappa)$ с ростом $\kappa$ происходит из точки $\lambda=0$ при тех и только тех значениях параметра $\kappa$, для которых $\kappa c \in \mathscr{E}$.

\title{
ЛИТЕРАТУРА
}

1. Шихов С. Б. Вопросы математической теории реакторов. Линейный анализ. Атомиздат, M., 1973. 2. Pихтмайер P. Принципы современной математической физики. Мир, M., 1982. 3. Lehner J., Wing G. M. Comm. Pure Appl. Math., 8, 217-234 (1955). 4. Lehner J. J. Math. Mech., 11, No. 2, 173-181 (1962). 5. Куперин Ю. А., Набоко C. Н., Романов Р. В. Функц. анализ и его прил., 33, вып. 3, 47-58 (1999). 6. Simon B. Functional integration and quantum physics. Academic Press, New York, 1979.

Московский государственный университет, механико-математический факультет

Поступило в редакцию 6 января 2000 г.

УДК 517.919

\section{Некристаллографические группы Кокстера и краевые особенности}

\author{
(C) 2001. И. Г. ЩЕРБАК
}

о. Введение. В работе 1972 г. [1] В. И. Арнольд описал деформации голоморфных ростков функций, миниверсальные относительно стабильной эквивалентности, и показал, что для ростков типа $A_{\mu}, D_{\mu}, E_{6}, E_{7}, E_{8}$ соответствующие бифуркационные диаграммы диффеоморфны многообразиям нерегулярных орбит комплексифицированного действия соответствующих групп отражений.

Деформации тех же ростков, миниверсальные относительно других отношений эквивалентности, возникают естественным образом при изучении особенностей с дополнительными структурами, такими, как граница, препятствие, симметрии и т. д. Среди соответствующих бифуркационных диаграмм можно распознать многообразия нерегулярных орбит других групп Кокстера. Так, например, миниверсальные деформации простых особенностей функций на многообразии с краем отождествляются с деформациями ростков $A_{2 \mu-1}, D_{\mu+1}, E_{6}$, миниверсальными в классе $\mathbb{Z}_{2}$-симметричных функций, и соответствующие бифуркационные диаграммы диффеоморфны многообразиям нерегулярных орбит групп отражений $B_{\mu}, C_{\mu}, F_{4}$ [2]. Многообразия нерегулярных орбит групп Кокстера $I_{2}(5), H_{3}$, $H_{4}$ появляются в задаче об обходе препятствия как бифуркационные диаграммы деформаций особенностей $A_{4}, D_{6}, E_{8}$ соответственно, миниверсальных в классе четнократных особенностей [3].

В отличие от этих примеров, особенности, связанные с группами Кокстера $I_{2}(p)(p \geqslant 5)$ и $H_{3}$, появляются в теории Ляшко критических точек функций на многообразии с особым краем [4] в ином контексте. В настоящей заметке устанавливается связь особенностей Ляшко с краевыми особенностями. Многообразия нерегулярных орбит групп $I_{2}(p)$ и $H_{3}$ оказываются диффеоморфными бифуркационным диаграммам краевых деформаций простых особенностей $A_{3}$ и $A_{4}$, связанных с краевыми особенностями $B_{p-1}^{3}$ и $F_{4}^{4}$ соответственно. Таким образом, эти группы включаются в «стандартную» схему, описанную выше. Необходимые понятия и факты читатель может найти в работах $[2,4,5]$ (см. также книгу $[6]$ ). 


\section{1. Функции на многообразии с особым краем и краевые особенности.}

В работе Ляшко [4] изучаются ростки функций на многообразии с особым краем, т. е. тройки $\left(f, V, \mathbb{C}^{n}\right)$, где $f:\left(\mathbb{C}^{n}, O\right) \rightarrow(\mathbb{C}, 0)-$ голоморфный росток, а $V=\left\{z \in \mathbb{C}^{n} \mid g(z)=0\right\}-$ росток гиперповерхности в изолированной простой особой точке $O$. Критические точки таких троек классифицируются с точностью до стабильной әквивалентности, т. е. до диффеоморфизмов пространства $\left(\mathbb{C}^{n}, O\right)$ и стабилизации, состоящей в добавлении квадратов новых переменных к уравнению края. Как отмечено в [4], если число переменных больше двух, то простые и унимодальные критические точки могут появляться только у неособых троек, т.е. таких, что 1-струя функции $f$ в $O$ ненулевая. В этом случае $g$ можно рассматривать как росток в простой критической точке $O$ функции на многообразии $\mathbb{C}^{n}$ с гладким краем $\{f(z)=0\}$.

ПредлОЖЕНИЕ. (1) Стабильно эквивалентные неособые тройки определяют одну и ту же краевую особенность.

(2) Простые критические точки троек отвечают простым краевым особенностям типа $C_{k}(k \geqslant 3), F_{4}$.

(3) Некритические точки троек отвечают простым краевым особенностям muna $B_{k}(k \geqslant 2)$.

В классификации Ляшко унимодальная критическая точка типа $I_{2}(p)(p \geqslant 5)$ задается ростком $f=x+\varepsilon y+z^{2}$ на гиперповерхности $x y=z^{p}$ (здесь $\varepsilon \neq 0-$ параметр вдоль страта $\mu=\mathrm{const})$. Легко проверить, что соответствующая краевая особенность имеет разложение $\left(A_{p-1}, A_{3}\right)$, и, значит, это особенность $B_{p-1}^{3}$ (в обозначениях работы [5]). Унимодальная критическая точка типа $H_{3}$ задается ростком $x+y+\varepsilon z^{3}$ на гиперповерхности $x y=z^{5}$ (снова $\varepsilon-$ параметр вдоль страта $\mu=$ const), а соответствующая краевая особенность имеет разложение $\left(A_{4}, A_{4}\right)$, т. е. это особенность $F_{4}^{4}$ в обозначениях из [5].

2. Локальные кольца и кратности. Оказывается, что во многих случаях локальное кольцио неособой тройки $\left(f, g(z)=0, \mathbb{C}^{n}\right)$ изоморфно локальному кольцу особенности $\left.g\right|_{f=0}$. Такие тройки мы называем хорошими. В частности, хорошими являются все тройки из списка Ляшко простых и унимодальных критических точек, тройки вида $\left(x, g\left(x, y_{1}, \ldots, y_{n-1}\right)=0, \mathbb{C}^{n}\right)$ с квазиоднородной функцией $g$ и тройки, отвечающие краевым особенностям с разложением $\left(A_{k}, A_{l}\right)$. Нам не известен пример нехорошей тройки с изолированной конечнократной критической точкой, но общий факт доказать не удалось; в каждом из перечисленных случаев доказательство свое.

Кратность критической точки хорошей тройки $\left(f, g(z)=0, \mathbb{C}^{n}\right)$ на 1 меньше кратности особенности $\left.g\right|_{f=0}$.

\section{3. Версальные деформации и бифуркационные диаграммы. Рассмотрим} росток $g(x, y)$ в точке $O$ пространства $\mathbb{C}^{n}$ с координатами $x, y=\left(y_{1}, \ldots, y_{n-1}\right)$ и краем $\{x=0\}$. Пусть $\left\{e_{i}=e_{i}(y), 0 \leqslant i \leqslant \mu\right\}-$ представители базиса локального кольца ростка $\left.g\right|_{x=0}=g(0, y)$. Назовем семейство

$$
G^{0}(y, \lambda)=g\left(\lambda_{0} e_{0}+\cdots+\lambda_{\mu} e_{\mu}, y\right)
$$

краевой деформациией ростка $\left.g\right|_{x=0}$, а соответствующую бифуркационную диаграмму

$$
\Sigma\left(G^{0}\right)=\left\{\lambda \in \mathbb{C}^{\mu+1} \mid 0-\text { критическое значение семейства } G^{0}(\cdot, \lambda)\right\}
$$


краевой бифуркационной диаграммой особенности $\left.g\right|_{x=0}$. Легко проверить, что эквивалентные краевые ростки задают диффеоморфные диаграммы.

Пусть теперь $x, y_{1}, \ldots, y_{n-1}-$ координаты в $\mathbb{C}^{n}$, в которых хорошая тройка имеет вид $L=\left(x, g(x, y)=0, \mathbb{C}^{n}\right)$. Согласно п. 2 , ее версальную деформацию можно взять в виде

$$
L_{\lambda}=\left(x+\lambda_{0} e_{0}+\cdots+\lambda_{\mu} e_{\mu}, g(x, y)=0, \mathbb{C}^{n}\right) .
$$

Этой деформации отвечает семейство краевых ростков, ограничение которого на край является краевой деформацией ростка $\left.g\right|_{x=0}$.

ТЕорема 1. Бифуркационная диаграмма хорошей тройки является краевой бифуркационной диаграммой соответствующей краевой особенности.

В частности, для тройки $\left(x, x^{2}+y^{5}=0, \mathbb{C}^{2}\right)$ типа $H_{3}$ соответствующая краевая деформация - семейство

$$
G_{H}^{0}(y, \lambda)=\left(\lambda_{3} y^{3}+\lambda_{2} y^{2}+\lambda_{1} y+\lambda_{0}\right)^{2}+y^{5},
$$

и бифуркационная диаграмма этой критической точки является краевой бифуркационной диаграммой особенности $A_{4}$, отвечающей краевой особенности $F_{4}^{4}$.

Критическая точка типа $I_{2}(p), p \geqslant 5$, может быть задана тройкой $\left(x,\left(x+y^{2}\right)^{2}+\right.$ $\left.y^{p}=0, \mathbb{C}^{2}\right)$. Соответствующая краевая деформация - семейство

$$
G_{I}^{0}(y, \lambda)=\left(y^{2}+\lambda_{2} y^{2}+\lambda_{1} y+\lambda_{0}\right)^{2}+y^{p},
$$

и соответствующая бифуркационная диаграмма - краевая бифуркационная диаграмма особенности $A_{3}$, отвечающая краевой особенности $B_{p-1}^{3}$.

Как показано в [4], бифуркационная диаграмма критической точки типа $\mathrm{H}_{3}$ (соответственно $I_{2}(p)$ ) аналитически тривиальна вдоль страта $\mu=$ const и $\lambda_{3}$ (соответственно $\lambda_{2}$ ) - параметр вдоль этого страта.

Теорема 2. Краевая бифуркационная диаграмма особенности $A_{4}$ (соответственно $A_{3}$ ), отвечающая краевой особенности $F_{4}^{4}$ (соответственно $B_{p-1}^{3}$ ), диффеоморфна многообразию нерегулярных орбит группы $H_{3}$ (соответственно $\left.I_{2}(p)\right)$, умноженному на комплексную прямую.

\section{ЛИТЕРАТУРА}

1. Арнольд В. И. Функц. анализ и его прил., 6, вып. 4, 3-25 (1972). 2. Арнольд В. И. УМН, 33, вып. 5, 91-105 (1978). 3. Щербак О. П. УМН, 43, вып. 3, 125-160 (1988). 4. Ляшко О. В. Функц. анализ и его прил., 17, вып. 3, 28-36 (1983). 5. Щербак И. Г. Краевые особенности с простым разложением. Труды семинара им. И. Г. Петровского, вып. 15, 1991, с. 55-69. 6. Арнольд В. И., Васильев Б. А., Горюнов В. В., Ляшко О. В. Особенности. II. Классификация и приложения. Итоги науки и техники. Современные проблемы математики. Фундаментальные направления, т. 39, ВИНИТИ, 1989.

Тель-Авивский университет 